\title{
Green computing and energy storage systems
}

\author{
Monika Żygadło ${ }^{1,}{ }^{*}$, Jerzy Kotowski ${ }^{1}$, and Jacek $\mathrm{Oko}^{2}$ \\ ${ }^{1}$ Wroclaw University of Science and Technology, Faculty of Electronics, 11/17 \\ Janiszewskiego st. 50-372 Wroclaw, Poland \\ ${ }^{2}$ Vice Chancellor for Informatization and the Director of the Centre for Networking and \\ Supercomputing at WrUST
}

\begin{abstract}
Because of growing amount of the data(known as ,,big data”) needed to be store and process there is a rapid growth of usage electricity and emission. In this paper we present the IT solution of this problem: green computing, solutions to storage and manage the energy and IT support needed to manage and control those systems. The article presents the main idea and already implemented solutions.
\end{abstract}

\section{Introduction}

In modern society energy consumption is continuing to grow. Non-renewable resources which use to be a primary source of energy are depleted. This leads to finding new ways of obtaining and saving the energy. It becomes important to accommodate and implement these solutions wherever possible. Nowadays IT is present in all branches of technology and science so one versatile system, easy to adapt for different needs, might save plenty of energy. The blend of green computing and energy storage systems (ESS) seems promising because of reduction of emissions, energy consumption and use of non-renewable energy sources at the same time. Green computing is not just about ecology but also saving money and resources. Data centers are essential elements of modern world, there are necessary to processing and store big data. A problem of data centers is high demand of power required to run the actual equipment and to cool the equipment. The solution is green data centers where the mechanical, lighting, electrical and computer systems are designed for maximum energy efficiency and minimum environmental impact. The construction and operation of a green data center includes advanced technologies and strategies. I.T industry is investing lot of time and money to devise new and effective ways to conserve energy. Companies like IBM, Hewlett Packard, SprayCool, and Cooligy are working on technologies such as liquid cooling, nano fluid-cooling systems, and in-server, in-rack, and in-row cooling. Other novel ways of making a data center more environmentally friendly include using new high-density servers, using hydrogen fuel cells as alternative green power sources, and applying virtualization technologies that reduce the total power consumption of servers and lower the heat generated.

\footnotetext{
*Corresponding author: monika.n.zygadlo@gmail.com
} 


\section{Green computing}

Green computing, Green ICT as per IFG International Federation of Green ICT and IFG Standard, green IT, or ICT sustainability, is the study and practice of environmentally sustainable computing or IT [1].

Murugesan [2] lays out the following four paths along which he believes the environmental effects of computing should be addressed:

1. Green Use: Reducing the energy consumption of computers and other information systems as well as using them in an environmentally sound manner.

2. Green Disposal: Refurbishing and reusing old computers and recycling unwanted computers and other electronic equipment.

3. Green Design: Designing energy efficient and environmentally sound components, computers, servers and cooling equipments.

4. Green Manufacturing: Manufacturing electronic components, computers and other associated sub systems with minimal impact or no impact on the environment.

Green computing is all about the efficient use of computers and computing [3]. The goals of green computing are quite similar to green chemistry which are to reduce the use of hazardous materials, maximize energy efficiency during the product's lifetime, and promote the recyclability or biodegradability of non-operational products and factory waste [1]. Green computing initiatives reduce the environmental impact of IT operations.

The following factors are impacting data centers as well as desktop computers, though to a lesser degree, and driving the need to adopt green computing practices [4]:

1. Rapid Growth Of The Internet: More and more people are increasingly relying on electronic data. There has been a rapid adoption of internet communications and media, computerization of business processes and applications, legal requirements for record retentions and disaster recovery. All these have led to the rapid growth in the size and number of data centers. On an individual level video and music downloads, on-line gaming, social networking site visits and VoIP are key drivers. Industry is also using internet increasingly. Internet usage is growing at more than 10 percent annually leading to an estimated $20 \%$ CAGR in data center demand.Disaster recovery strategies that emphasizes on maintaining duplicate records increases demand further. Many federal, state, and local government agencies have adopted e-government strategies that utilize the Web for public information, reporting, transactions, homeland security, and scientific computing.

2. Increasing Equipment Power Density: Although advances in server CPUs have in some cases enabled higher performance with less power consumption per CPU, overall server power consumption has continued to increase as more servers are installed with higher performance power-hungry processors with more memory capacity. As more servers are installed they require more floor space. To pack more servers in the same footprint the form factor of servers has become much smaller, in some cases shrinking by more than $70 \%$ through the use of blade servers. This increase in packaging density has been matched by a major increase in the power density of data centers. Density has increased more than ten times from 300 watts per square foot in 1996 to over 4,000 watts per square foot in 2007, a trend that is expected to continue its upward spiral.

3. Increasing Cooling Requirements: The increase in server power density has led to a associated increase in data center heat density. Servers require approximately 1 to 1.5 watts of cooling for each watt of power used. The ratio of cooling power to server power requirements will continue to increase as data center server densities increase.

4. Increasing Energy Costs: Data center expenditures for power and cooling can exceed that for equipment over the useful life of a server. One study estimated that for a typical $\$ 4,000$ server rated at 500 watts, it would consume approximately $\$ 4,000$ of electricity for power 
and cooling over three years, at $\$ 0.08$ per kilowatt-hour, and double that in Japan. The ratio of power and cooling expense to equipment expenses have increased from approximately 0.1 to 1 in 2000 to 1 to 1 in 2007 . With the likely increase in the number of data centers and servers and the advent of a carbon cap-and-trade scheme, the cost of energy for data center power and cooling will increase continuously.

5. Restrictions On Energy Supply And Access: Companies such as Google, Microsoft, and Yahoo with the need for large data centers may not be able to find power at any price in major American cities. Therefore, they have built new data centers in the Pacific Northwest near the Columbia River where they have direct access to low-cost hydroelectric power and this has proved beneficial to them because they do not depend on the overtaxed electrical grid. In states such as, California, Illinois, and New York, the old electrical infrastructure and high costs of power can stall or stop the construction of new data centers and limit the operations of existing centers. In some crowded urban areas utility power feeds are at capacity and electricity is not available for new data centers at any price.

6. Low Server Utilization Rates: Data center efficiency is a major problem in terms of energy use. The server utilization rates average 5-10 per cent for large data centers. Low server utilization means companies are overpaying for energy, maintenance, operations support , while only using a small percentage of computing capacity.

7. Growing Awareness Of I.T's Impact On The Environment: Carbon emissions are directly proportional to energy usage. In 2007 there were approximately 44 million servers worldwide consuming $0.5 \%$ of all electricity. Data centers in the U.S use more than $1 \%$ of all electricity. Their collective annual carbon emissions of 80 metric megatons of $\mathrm{CO}_{2}$ are approaching the carbon footprint of the Netherlands and Argentina. Carbon emissions from operations are expected to grow at more than $11 \%$ per year to 340 metric megatons by 2020 . Additionally, the carbon footprint of manufacturing the IT product is largely unaccounted for by IT organizations.

The continued popularity and consequent rise of Internet and Web applications is driving the rapid growth of data centers. Enterprises are installing more servers or expanding their capacity to address to the ever increasing demand of electronic data. The number of server computers in data centers has increased six fold to 30 million in the last decade, and each server draws far more electricity than earlier models. Combined electricity use for servers doubled between 2000 and 2005, most of which came from businesses installing large numbers of new servers. The operational cost of data centers continues to increase steadily with ever increasing energy prices worldwide. Besides the cost, availability of electrical power is becoming a critical issue for many companies whose data centers have expanded steadily. Constraints like social, financial, and practical is forcing businesses and IT departments to reduce energy consumption by data centers. Data center efficiency can be improved by using new energy-efficient equipment, improving airflow management to reduce cooling requirements, investing in energy management software, and adopting environmentally friendly designs for data centers and adopting new measures to curb data centers energy consumption [2]. So proper management of data centers is the need of the hour.

Cloud computing is good example of implementation the idea of green computing. Cloud infrastructure addresses two critical elements of a green IT approach: energy efficiency and resource efficiency(Fig. 1). The broad scope of cloud computing is succinctly summarized in [5]: Cloud computing is a model for enabling convenient, on-demand network access to a shared pool of configurable computing resources that can be rapidly pro-visioned and released with minimal management effort or service provider interaction.

Cloud computing become common in big data processing. It is more efficient, faster and cheaper than traditional infrastructure. 


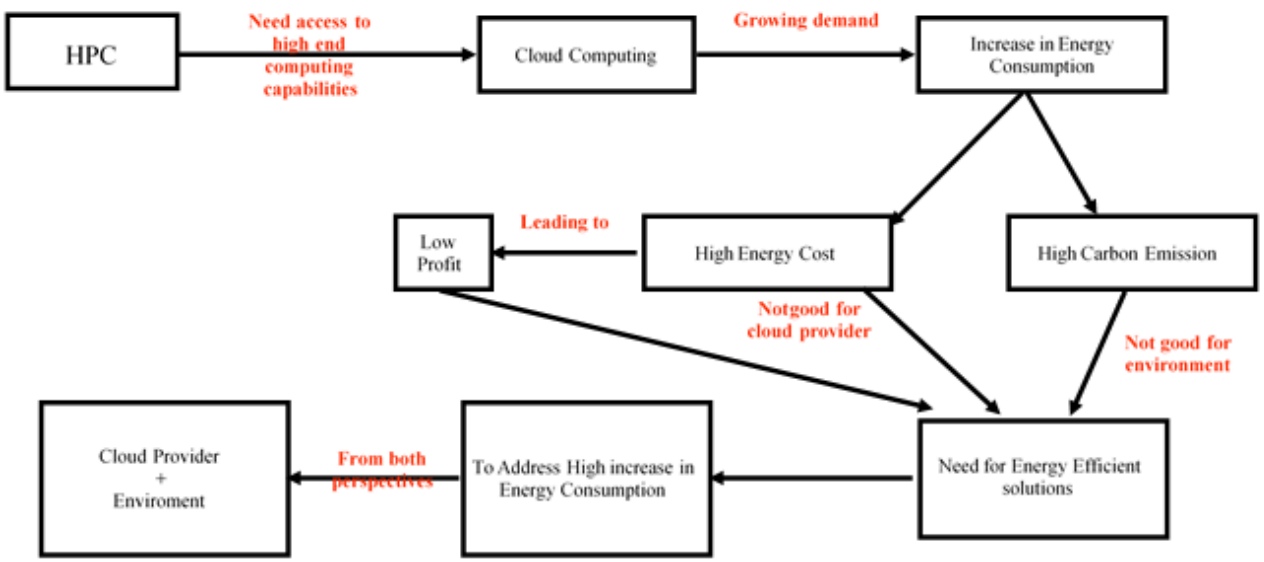

Fig. 1. Green Computing in Clouds.

\section{Energy storage systems}

Electrical Energy Storage (EES) refers to a process of converting electrical energy from a power network into a form that can be stored for converting back to electrical energy when needed. Such a process enables electricity to be produced at times of either low demand, low generation cost or from intermittent energy sources and to be used at times of high demand, high generation cost or when no other generation means is available. EES has numerous applications including portable devices, transport vehicles and stationary energy resources [6]. Energy storage can bring about a reduction in operating costs or capital expenditures when used as a generation resource in the utility sector. When used with renewable resources, energy storage can increase their usability of photovoltaic and wind generated electricity by making this generation coincident with peak load demand. Energy storage may facilitate the inclusion of wind and solar energy into the electric grid (Fig. 2).

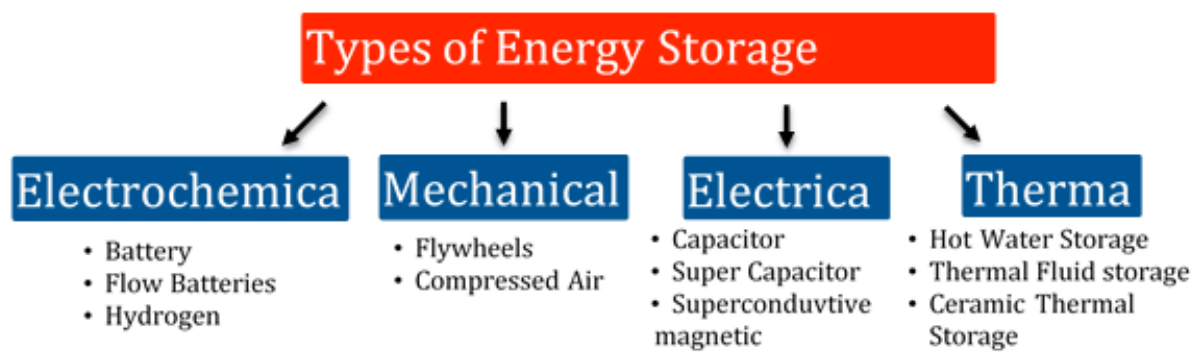

Fig. 2. Types of Energy Storage Systems.

When EES is adopted for bridging power, they are required to have moderate power rating $(100 \mathrm{~kW}-10 \mathrm{MW}$ ) and response time (up to around $1 \mathrm{~s}$ ), in order to provide the continuity of power supply at energy gap periods (up to several hours), such as the time interval for switching the system from one source of power generation to another(Fig. 3). Conventional batteries and flow batteries are suitable for this application. Flywheels, supercapacitors and fuel cells are also reported for such types of applications. Piller Power Systems Ltd. has practical experiences in using flywheels as ride-through power sources. EES plays an important role in energy management for optimizing energy uses and decoupling the timing 
of generation and consumption of electric energy. Time shifting and peak shaving are typical applications in energy management. Energy management application can be further classified based on the application power rating: large-scale (above $100 \mathrm{MW}$ ) and medium/small-scale ( 1-100 MW). PHS, large-scale CAES and TES can be used for large-scale energy management. Flow batteries, large-scale conventional batteries, fuel cells and solar fuels are more suitable for small/medium-scale energy management [7].

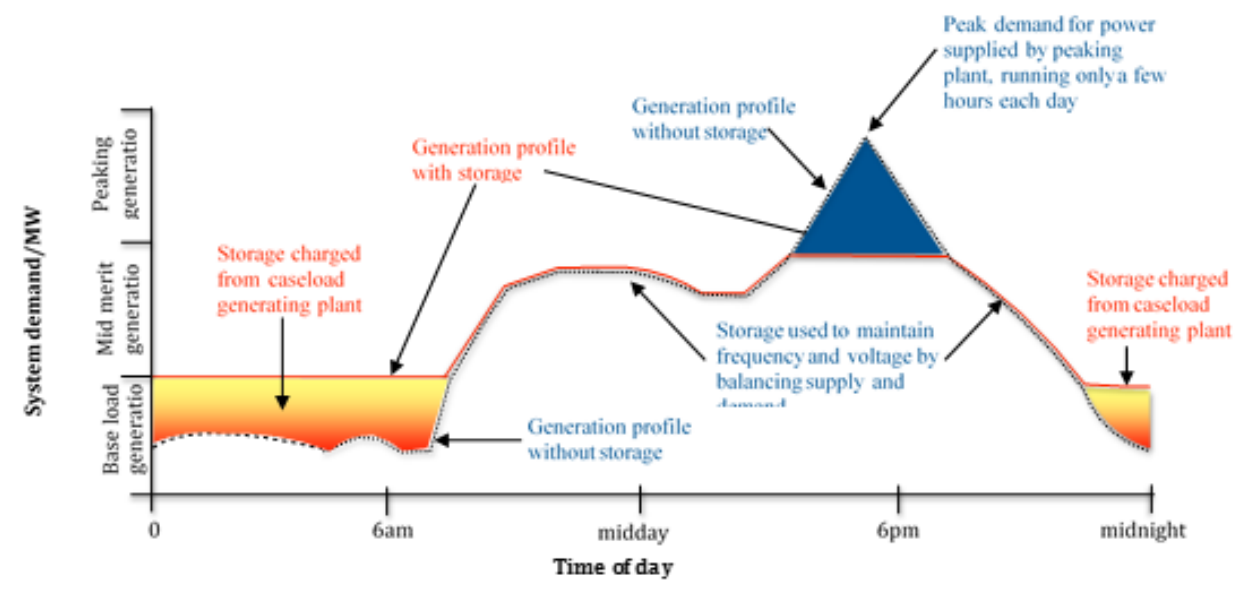

Fig. 3. EES time lapse.

The graph explains that the system demand can be handled efficiently if storage is incorporated into the electrical network. As shown the storage is charged from the base load generating plant during the early hours of the day when the demand is low. And then as the demand rises during the day the generating plants belonging to mid merit category, account for the demand. And during the peak demand time if storage is taken into account then the demand can be supplied by the peaking plant which runs only for few hours of the day decreasing the total cost of operating such a storage incorporated system. Thus we see that when the generation profile with storage is taken, there is a much controlled demand graph as storage takes care of the load leveling and then it is charged again at the end of the day from the base load generating plant [8].

The biggest ESS in Poland will be built in norther part of the country. The project consists of Special Protection Scheme (SPS) and Hybrid Battery Energy Storage System (BESS) equipped with lithium-ion and lead-acid batteries for wind power generation will have capacity of $27 \mathrm{MWh}$ [9]. An SPS system plans countermeasures actions for specific accidents on the power network based on online information extracted from SCADA. If an accident actually occurs, the SPS system carries out controls automatically in order to prevent overloads in the power system.

It can be seen that the current technologies have wide ranging technological characteristics. With a suitable combination of different technologies, EES can meet most technical requirements for different power system and network operations. However, apart from PHS, most EES technologies are not cost-effective or mature enough for widespread implementation within the current large network operation regulation and energy market frame. On the other hand, the benefits brought to power system operation by utilizing EES technologies need further exploration. The capital and the maintenance cost of an EES system varies with the timescale of construction, the location of the facility, the size of the system, the material chosen for storing energy (such as PCMs in TES) and many other factors. Although a number of demonstration projects or EES trial stations were completed, the corresponding detailed techno-economic analysis, which can enhance the relevant database 
to practical EES experience, is still not sufficient. The widespread deployment of EES will depend on advances in relevant technologies, but it also relies on progress in further quantification and analysis of the benefits brought by EES [7].

\section{Scientific and technical challenges}

The Wroclaw University of Science and Technology is currently one of the biggest and best universities in Poland. The university is a regional leader in the industry and innovation sector, which is confirmed by dozens of agreements signed with companies of international renown. Constant efforts to build co-operation network with local, regional and international partners allow the information flow between the sectors of science, regional governments and enterprises, this in turn results in sharing knowledge and experience which is beneficial for the regional potential growth.

The Wroclaw University of Science and Technology laboratories use an extensive scientific basis and technical resource base equipped with unique apparatus and that is why they are able to conduct complex research procedures including the analysis of results, the design process and making prototypes. Among others, they are authorized to calibrate equipment, offer research and services related to EU standards of conformity assessment of products, systems and installations.

In the summer of 2010, IBM Poland and Wroclaw University of Technology introduced the first University Cloud Computing Centre in Poland, a Centre that is funded by IBM's Multipurpose Cloud Computing Centre program. Wroclaw University of Technology is the first University worldwide to collaborate with IBM for this initiative. The Centre aspires to use the Cloud for education, to use the Cloud for a remote educational internship, and to promote research through in Cloud technologies and to offer services to other faculties and business partners. Due to this cooperation now WruST is equipped with the special computer named Smartcloud Orchestrator. It is the first implementation in the world, designed to improve an education processes and research. Orchestrator is a workflow management solution for the data center. Orchestrator lets to automate the creation, monitoring, and deployment of resources. Cloud orchestration solutions from IBM are designed to reduce the IT management complexities introduced by virtual and cloud environments and accelerate cloud service delivery, allowing enterprises to quickly respond to changing business needs. IT administrators perform many tasks and procedures to keep the health of their computing environment up-to-date and their business running. Tasks might include the diverse activities. Individual tasks and subtasks are automated, but typically, not for the whole process.

By using Orchestrator, we can carry out the following tasks:

- Automate processes in the data center, regardless of hardware or platform,

- automate IT operations and standardize best practices to improve operational efficiency and connect different systems from different vendors without having to know how to use scripting and programming languages.

- Orchestrator provides tools to build, test, debug, deploy, and manage automation in environment. The standard activities defined in every installation of Orchestrator provide a variety of monitors and tasks with which it is possible to integrate a wide range of system processes. Any IT organization can use Orchestrator to improve efficiency and reduce operational costs to support cross-departmental objectives. Orchestrator provides an environment with shared access to common data. By using Orchestrator, an enterprise can evolve and automate key processes between groups and consolidate repetitive manual tasks. It can automate cross-functional team processes and enforce best practices for incident, change, and service management. All the changes in research will not be possible without a flexible system that would support cooperation between all types of schools and interactions with businesses. IBM Smarter Cloud Orchestrator (SCO) at WrUST is a base on which such 
systems for the entire region of Lower-Silesia will be built. The first phase of the project consists of implementation of an IT Cloud for education at the University with the architecture and technology already prepared to support future extension.

The system at the WrUST contains the virtual platform of the system based on four servers IBM Flex System x240. At WrUST System SmartCloud Orchestrator was installed on virtual machines with operational system Red Hat Enterprise Linux 6.3 x64. The components of Cloud System are as follows:

- Smart Cloud Orchestrator,

- IBM Image Construction and Composition Tool,

- IBM Virtual Image Library,

- IBM Process Center,

- $\quad$ IBM Process Portal [10].

Good IT support provides to full use of possibilities which gives green computing. Well implemented green computing rules combined with EES and IT support enable to create ecological, efficient and economic system. IT support is responsible for optimal usage of energy and computing power, but to do that in proper way the equipment and software have to be chosen accordingly to required effects.

\section{References}

1. http://en.wikipedia.org/wiki/Green_computing

2. S. Murugesan, IT professional 10.1, 24-33 (2008)

3. S. Mishra, Green Computing, Science Horizon, 21 (2013)

4. R. Harmon, N. Auseklis, Sustainable IT services: Assessing the impact of green computing practices, Management of Engineering \& Technology, Portland International Conference on. IEEE (2009)

5. P. Mell, T. Grance, The NIST Definition of Cloud Computing, National Institute of Standards and Technology (2011)

6. H. Chen, T. Ngoc Cong, W. Yang, C. Tan, Y. Li, Y. Ding, Progress in electrical energy storage system: A critical review, Progress in Natural Science, 19, 291-312 (2009)

7. X. Luo, J. Wang, M. Dooner, J. Clarke, Applied Energy, 137, 511-536 (2015)

8. A. Joseph, M. Shahidehpour, Battery storage systems in electric power systems, ECE Department, Illinois Institute of Technology Chicago, Illinois, USA

9. https://media.energa.pl/pr/358081/na-farmie-wiatrowej-energi-powstanie-najwiekszyw-polsce-magazyn-energii?rss=true

10. J. Greblicki, J. Kotowski, M, Ochla, J.Oko, SmartCloud Orchestrator-The First Implementation for Education in the world at WrUT, Computational Intelligence and Efficiency in Engineering Systems, 209-215 (2015) 